\title{
Dermatofibrosarcoma Protuberans
}

National Cancer Institute

\section{Source}

National Cancer Institute. Dermatofibrosarcoma Protuberans. NCI Thesaurus. Code C4683.

A low grade fibroblastic neoplasm presenting as a nodular cutaneous mass, most often on the trunk and the proximal extremities. The tumor diffusely infiltrates the dermis and the subcutaneous tissues. It is considered a locally aggressive neoplasm, which often recurs but rarely metastasizes. 